\title{
Managing direct oral anticoagulants in patients undergoing dentoalveolar surgery
}

\author{
J. P. Patel, ${ }^{* 1,2}$ S. A. Woolcombe, ${ }^{3}$ R. K. Patel, ${ }^{1}$ O. Obisesan, ${ }^{3}$ L. N. Roberts, ${ }^{1}$ C. Bryant ${ }^{3}$ and R. Arya ${ }^{1}$
}

In brief

Describes the first UK evaluation of undertaking

dental extractions in patients prescribed DOACs.

Provides useful information on direct oral

anticoagulants for other practitioners.

Key considerations are the time of last DOAC dose in relation to the dentoalveolar procedure and time of

DOAC recommencement post-procedure.

Our objective was to describe our experience of managing a cohort of adult patients prescribed direct oral anticoagulants (DOACs) undergoing dentoalveolar procedures between November 2012 and May 2016. Prior to conducting a procedure a formal assessment was made of each patient's anticoagulation treatment. A specific plan was then formulated, balancing the risk of bleeding with the risk of thrombosis. Patients received a telephone consultation one week following treatment to assess any post-operative bleeding. Eighty-two patients underwent 111 oral surgical procedures, the majority of which were dental extractions. In the case of $35(32 \%)$ procedures, advice was given to omit the DOAC, either before or after treatment. There was no bleeding following the majority of procedures. Persistent bleeding followed 15 (13.5\%) procedures, of which 7 (6.3\%) procedures required specific intervention. The majority of patients prescribed DOACs can undergo dentoalveolar procedures safely. Important considerations when planning treatment are: (i) when the patient usually takes their dose of DOAC, (ii) the time the procedure is performed and, (iii) when the DOAC is taken post-procedure. In our experience, if these factors are considered carefully, omission of DOAC doses is unlikely to be required for most patients.

\section{Introduction}

It is relatively common for patients who are anticoagulated to require dentoalveolar surgery, particularly dental extractions. In the RELY study, ${ }^{1} 460$ patients ( $2.5 \%$ of study cohort) required a dental procedure during the course of the trial. The risk of persistent bleeding complicating dental extraction in patients who are not receiving oral anticoagulants is estimated to be $1 \%{ }^{2,3}$ In patients who are prescribed oral anticoagulants this risk is reported to increase to between $4 \%$ and $9 \%{ }^{2,4}$

In order to overcome inconsistencies that patients prescribed vitamin $\mathrm{K}$ antagonists (VKAs) experienced while undergoing dentoalveolar procedures, Perry et al. ${ }^{5}$ compiled

${ }^{1}$ Department of Haematological Medicine; ${ }^{3}$ Department of Oral Surgery, King's College Hospital NHS Foundation Trust; 2Institute of Pharmaceutical Science, King's College London ${ }^{*}$ Correspondence to: Jignesh P. Patel

Email: jig.patel@kcl.ac.uk

Refereed Paper. Accepted 3 January 2017

DOI: 10.1038/sj.bdj.2017.165

${ }^{\oplus}$ British Dental Journal 2017; 222: 245-249 and published guidelines, endorsed by the British Society of Haematology, which has led to a standardised approach to patient management within this setting. These guidelines state that the INR should be assessed within 72 hours before the procedure and if below 4 then usually no specific intervention is required. The guidelines also suggest that the risk of bleeding should be minimised by the use of local haemostatic measures and 5\% tranexamic acid mouthwash four times a day for 48 hours post-procedure.

In recent years, the anticoagulation landscape has changed with the availability of direct oral anticoagulants (DOACs), for example, apixaban, dabigatran, edoxaban and rivaroxaban. ${ }^{6}$ This raises the question of whether these agents require special management peri-procedurally. Given the current advice regarding oral VKA therapy, one might simply follow the course of action that is followed for VKAs, ie to advise the patient to continue as per usual and minimise the risk of bleeding with local haemostatic measures, as outlined previously. However, the DOACs have a rapid onset and offset of action relative to VKAs, ${ }^{7}$ which makes these agents different in a peri-procedural setting (Table 1). Additionally, measurement of anticoagulation intensity is not usually required or readily available for DOACs.

Based on the manufacturers' recommendations, one might consider it prudent to ask the patient to omit a dose of a DOAC before the dentoalveolar procedure, in order to reduce the risk of bleeding. The rapid onset of action of the DOACs also requires careful consideration. If a patient takes a dose of DOAC within four hours of completing a dentoalveolar procedure they could be exposed to high anticoagulant activity in the early post-operative period. This may potentially increase the risk of bleeding, with peak concentrations of DOACs being reached within four hours of ingesting a dose.

During the past four years, as individual DOACs have become available worldwide, information on their use in the real-world population has been growing. ${ }^{9-11}$ There is, however, little available information regarding patients having dentoalveolar procedures while 
Table 1 Key properties ${ }^{8}$ of the direct oral anticoagulants which are currently available for use in the UK

\begin{tabular}{|c|c|c|c|c|}
\hline $\begin{array}{l}\text { Pharmacokinetic } \\
\text { parameter }\end{array}$ & Dabigatran & Apixaban & Edoxaban & Rivaroxaban \\
\hline Bioavailability (\%) & 6 & 70 & 62 & 80 \\
\hline Peak onset (h) & $1.5-2$ & $3-4$ & $1-2$ & $2-4$ \\
\hline Elimination half-life $(h)^{*}$ & $12-14$ & 12 & $10-14$ & $7-11$ \\
\hline $\begin{array}{l}\text { Manufacturers' } \\
\text { recommendations on } \\
\text { discontinuation before } \\
\text { surgical procedure }\end{array}$ & $\begin{array}{l}\text { If possible, dabigatran should be discontinued } \\
\text { at least } 24 \text { hours before invasive or surgical } \\
\text { procedures. In patients at higher risk of } \\
\text { bleeding or in major surgery where complete } \\
\text { haemostasis may be required, consider } \\
\text { stopping dabigatran } 2-4 \text { days before surgery. } \\
\text { Clearance of dabigatran in patients with renal } \\
\text { insufficiency may take longer. This should be }\end{array}$ & $\begin{array}{l}\text { Apixaban should be stopped } \\
48 \text { hours before a procedure } \\
\text { with a moderate or high risk } \\
\text { of bleeding and } 24 \text { hours } \\
\text { before a procedure with a } \\
\text { low risk of bleeding. }\end{array}$ & $\begin{array}{l}\text { If anticoagulation must } \\
\text { be discontinued to reduce } \\
\text { the risk of bleeding with } \\
\text { surgical or other procedures, } \\
\text { edoxaban should be stopped } \\
\text { as soon as possible and } \\
\text { preferably at least } 24 \text { hours } \\
\text { before the procedure. }\end{array}$ & $\begin{array}{l}\text { If an invasive procedure } \\
\text { or surgical intervention is } \\
\text { required, rivaroxaban } 20 \mathrm{mg} \\
\text { should be stopped at least } \\
24 \text { hours before the interven- } \\
\text { tion, if possible. }\end{array}$ \\
\hline $\begin{array}{l}\text { Manufacturers' } \\
\text { recommendations on } \\
\text { restarting post-surgical } \\
\text { procedure }\end{array}$ & $\begin{array}{l}\text { Dabigatran etexilate should be restarted after } \\
\text { the invasive procedure or surgical interven- } \\
\text { tion as soon as possible provided the clinical } \\
\text { situation allows and adequate haemostasis } \\
\text { has been established. } \\
\text { Patients at risk for bleeding or patients at } \\
\text { risk of overexposure, notably patients with } \\
\text { moderate renal impairment (CrCL } 30-50 \mathrm{~mL} / \\
\text { min), should be treated with caution. }\end{array}$ & $\begin{array}{l}\text { Apixaban should be restarted } \\
\text { after the invasive procedure } \\
\text { or surgical intervention as } \\
\text { soon as possible provided the } \\
\text { clinical situation allows and } \\
\text { adequate haemostasis has } \\
\text { been established. }\end{array}$ & $\begin{array}{l}\text { Edoxaban should be restarted } \\
\text { after the surgical or other } \\
\text { procedures as soon as } \\
\text { adequate haemostasis has } \\
\text { been established, noting } \\
\text { that the time to onset of the } \\
\text { edoxaban anticoagulant ther- } \\
\text { apeutic effect is } 1-2 \text { hours. }\end{array}$ & $\begin{array}{l}\text { Rivaroxaban should be } \\
\text { restarted as soon as possible } \\
\text { after the invasive procedure } \\
\text { or surgical intervention } \\
\text { provided the clinical situation } \\
\text { allows and adequate haemo- } \\
\text { stasis has been established } \\
\text { as determined by the treating } \\
\text { physician. }\end{array}$ \\
\hline
\end{tabular}

taking these agents. Recently Douketis et al. ${ }^{12}$ reported the outcomes of patients who required a surgical intervention during the RELY trial. Four hundred and sixty patients in the study required a dental procedure, 455 elective procedures and five emergency procedures. The authors found that there were no differences in outcomes between the warfarin and dabigatran arms of the trial, although the risk of bleeding or thromboembolism was higher in patients having an urgent procedure compared to those patients having an elective procedure.

As the use of DOACs has become more prevalent in the UK, they have received increasing attention in the dental community. ${ }^{13-21}$ Early discussions focused on increasing the awareness of these agents, with more recent considerations concentrating on how to manage the agents peri-procedurally. The Scottish Dental Clinical Effectiveness Programme (SDCEP) published guidance on the management of dental patients taking anticoagulants or anti-platelet drugs in $2015 .{ }^{22}$ To the best of our knowledge, this is the only guidance which formally addresses how DOACs might be managed in this specific setting. The guidance suggests that if the bleeding risk is low, treatment should be performed without interrupting the DOAC and that if the bleeding risk is high (according to a list of specified procedures), patients should be advised to miss or delay the morning dose before treatment. For both low and high risk procedures the guidance suggests treating early in the day, limiting the initial treatment area and assessing bleeding before continuing, staging extensive or complex procedures and actively considering suturing and packing. It also stipulates that patients should be given specific advice on when it might be safe to restart their DOAC. Additionally, the guidance recommends consulting a general medical practitioner or specialist for patients who have other relevant medical complications which may further increase the risk of bleeding, including liver, renal and bone marrow disorders. This guidance has only recently become available. We however have been managing patients on DOACs for the past four years. Our paper describes our clinical experience of conducting oral surgical procedures in patients prescribed DOACs to date.

\section{Methods}

In our hospital, we began to prescribe DOAC therapy, according to licensed indications, in the summer of 2012. This followed NICE approval for dabigatran in the context of atrial fibrillation $(\mathrm{AF})^{23}$ in line with the South London Stroke and Cardiac Network guidance. ${ }^{24}$ We had no clinical experience of managing DOAC therapy during dentoalveolar surgery. Therefore the Department of Oral Surgery and the Department of Haematological Medicine decided to prospectively manage each patient on a case by case basis from November 2012, when the first patient prescribed a DOAC underwent an oral surgical procedure. This involved considering the patient's past medical history, the indication for anticoagulation, any renal impairment and the risk of bleeding associated with the procedure planned, along with the risk of thrombosis if anticoagulant therapy were to be temporarily stopped. In addition, patients had the anticoagulation intensity assessed (varying time after dose), before the procedure with a calibrated anti-Xa-assay for apixaban and rivaroxaban (STA-liquid anti-Xa assay [Diagnostica Stago, France]) and an ecarin time assay, calibrated for dabigatran (STA-ECA II dabigatran chromogenic assay [Diagnostica Stago, France]). The results were reported back in $\mathrm{ng} / \mathrm{mL}$ for the respective drug assayed. After consideration of the relevant information a specific plan was formulated for each patient, including whether the DOAC should be simply continued as per usual, omitted, or the timing of DOAC administration altered. Furthermore, as we had no clinical experience of DOAC use in this setting, we conducted a telephone review for each patient, one week following the oral surgical procedure, to elucidate if any delayed post-operative bleeding had occurred.

If a patient did experience bleeding, information regarding this was captured and categorised according to the following: (i) bleeding present, but no action required; (ii) required consultation in the dental unit, but no specific intervention required; (iii) required surgical 
intervention (resuturing and haemostatic packing) and/or use of an antifibrinolytic; and (iv) required blood transfusion, replacement therapy or desmopressin.

Information on all patients was retrospectively entered onto SPSS v20 and analysed. Descriptive statistics were used to analyse and present the results.

\section{Results}

During the time we have been collecting information, 92 patients have been referred for oral surgical procedures to the unit, of which 82 patients underwent 111 procedures while prescribed a DOAC. The ten remaining patients referred were excluded because three had discontinued DOAC therapy prior to the procedure, one was deemed medically unfit for the procedure and six did not attend for their planned treatment. Table 2 summarises the demographic information on the patients who did have a procedure while on DOAC therapy. In our unit the number of procedures conducted on patients prescribed concurrent DOAC has increased year on year, reflecting an increase in the use of DOACs in our locality. We undertook one procedure in 2012, 11 in 2013, 18 in 2014, 50 in 2015 and 31 at the time of writing in May 2016.

Forty-six patients (56.1\%) were prescribed DOAC for stroke prevention in the context of AF, 28 (34.1\%) were prescribed DOAC for the acute and secondary prevention of venous thromboembolism (VTE), four patients (4.9\%) were prescribed DOAC for other indications and in four cases (4.9\%), the indication was not clear. Seventy-five (91.5\%) patients had DOAC concentrations assayed before the procedure to assess anticoagulation intensity. Of these, 72 patients (96\%) had concentrations considered in range, in line with published data. Three patients (4\%) had concentrations suggestive of over-anticoagulation and in such cases advice was given to omit dosing of DOACs before the procedure, to account for this. Nine patients were prescribed concomitant anti-platelet therapy with their DOAC; three were prescribed aspirin, four were prescribed clopidogrel and two patients were prescribed a combination of clopidogrel and aspirin. Two patients were prescribed a regular non-steroidal anti-inflammatory drug (NSAID), ibuprofen and naproxen respectively. Twenty-one patients were prescribed a twice daily DOAC and the remaining 61 patients were taking rivaroxaban once daily. Twenty-eight patients took this in the
Table 2 Demographic information on the patients who underwent a dentoalveolar procedure \begin{tabular}{l|l} 
Demographic information $(\mathrm{N}=82)$ & $\mathrm{N}(\%)$ unless otherwise specified
\end{tabular}

\begin{tabular}{l|l}
\hline Age, years - mean (min-max) & $67.3(19-93)$ \\
\hline Female gender & $36(43.9)$ \\
\hline Height, $\mathrm{cm}(\mathrm{sd})$ & $168.4(9.95)$ \\
\hline Weight, $\mathrm{kg}(\mathrm{sd})$ & $86.6(23.3)$ \\
\hline Calculated $\mathrm{CrCl}{ }^{*}$ - mean $\mathrm{mL} / \mathrm{min}(\min -\mathrm{max})$ & $82(29-224)$ \\
\hline Concurrent anti- platelet prescribed & $9(10.9)$ \\
\hline Concurrent NSAID prescribed & $2(2.4)$ \\
\hline
\end{tabular}

\section{DOAC prescribed}

\begin{tabular}{l|l}
\hline Rivaroxaban $20 \mathrm{mg}$ od & $46(56)$ \\
\hline Rivaroxaban $15 \mathrm{mg}$ od & $15(18)$ \\
\hline Apixaban $5 \mathrm{mg}$ bd & $4(5)$ \\
\hline Apixaban $2.5 \mathrm{mg}$ bd & $7(9)$ \\
\hline Dabigatran $150 \mathrm{mg}$ bd & $2(2)$ \\
\hline Dabigatran $110 \mathrm{mg}$ bd & $7(9)$ \\
\hline Other DOAC dose (rivaroxaban $10 \mathrm{mg}$ bd) & $1(1)$ \\
\hline
\end{tabular}

*Based on adjusted body weight, when the patient was obese

Table 3 Procedure and anaesthesia type

Procedure conducted

$\mathrm{N}(\%)$

Type of procedure

\begin{tabular}{l|l}
\hline Routine dental extraction & $82(73.9)$ \\
\hline Surgical dental extraction & $23(20.7)$ \\
\hline Other $^{*}$ & $6(5.4)$
\end{tabular}

Single or multiple

\begin{tabular}{l|l}
\hline Single & $53(47.7)$ \\
\hline Multiple & $58(52.3)$ \\
\hline
\end{tabular}

\section{Anaesthesia type}

\begin{tabular}{l|l}
\hline Local infiltration only & $73(66)$ \\
\hline Inferior dental (ID) block & $38(34)$
\end{tabular}

${ }^{*}$ This was excisional biopsy $(\mathrm{x} 1$ ), surgical dental extraction and incision and drainage $(\mathrm{x} 1$ ), enucleation of cyst $(\mathrm{x} 1)$, routine extraction of upper left second premolar root and curettage of apical granulation tissue (x1), labial salivary gland biopsy (x1), extraction of upper left second premolar
incisional biopsy of buccal mucosa $(x 1)$

\section{Table 4 Specific advice given to patients before the procedure}

\begin{tabular}{l|l}
\hline Advice given & $\mathbf{n}(\%)$ \\
\hline Continue DOAC as per usual & $68(61)$ \\
\hline Omit a single dose before procedure & $26(23)$ \\
\hline Omit a single dose before and after procedure & $5(5)$ \\
\hline Omit a single dose after procedure & $3(3)$ \\
\hline Delay the evening dose after procedure & $8(7)$ \\
\hline Omit for 2 days before procedure and re-start the evening following procedure & $1(1)$ \\
\hline
\end{tabular}


morning, three in the afternoon and 30 patients took their rivaroxaban in the evening. The 111 procedures that the 82 patients underwent are summarised in Table 3.

In our cohort specific advice was given to omit doses of DOAC before and/or after the procedure for 35 (32\%) procedures, and for eight $(7 \%)$ procedures advice was given to delay the evening dose after the procedure. Table 4 describes in categories the advice given.

Interestingly, 14 patients (15 [13.5\%] procedures) did not follow the pre-operative advice and/or post-operative advice given, either omitting doses when advised to continue as usual or omitting more doses of DOAC than recommended.

Over time and with increasing experience, advice to omit doses of DOACs became less frequent and whenever possible, simply adjusting the timing of DOACs on the day of the procedure became more prevalent.

On the day of the procedure, the median time that had elapsed between the last dose of DOAC and the start of the procedure was 15 hours (inter-quartile range $8-20$ ). No patients experienced bleeding or haematoma at the site of inferior dental (ID) block injection.

Persistent bleeding occurred following 15 (13.5\%) procedures. For six procedures the bleeding was trivial and no specific action was required. Following two procedures specific consultation in the oral surgery unit was required but no intervention was implemented. Intervention to arrest bleeding, either surgical (resuturing and haemostatic packing) and/or use of an antifibrinolytic agent, was required following the remaining seven (6.3\%) procedures.

The details of the six patients who had bleeding requiring intervention are provided online as supplementary information Table 1.

\section{Discussion}

This paper describes the experience in our centre of managing patients prescribed DOACs as they underwent dentoalveolar procedures over the past four years. To the best of our knowledge, this is one of the first evaluations of using DOACs in a large cohort in this setting and provides a valuable insight into how best to manage patients undergoing these procedures.

Due to lack of guidance and practical experience regarding the impact of continuing DOACs peri-procedurally, our initial approach was to minimise drug exposure at the time of surgery. This involved omitting a dose/doses on the day of treatment, before and/or after the procedure, indication permitting. However, with increasing experience it became apparent that such a conservative approach was not required if the risk of bleeding was low. In such cases continuing the DOAC as per usual became the recommended management. Such an approach has recently been endorsed by the SDCEP guidance. The experience we have to date suggests that such advice is sensible and we now most often follow this approach for new patients referred for treatment, providing they do not have additional risk factors for bleeding.

In our cohort, post-operative bleeding followed 15 (13\%) procedures, of which six (5\%) procedures had minor or trivial bleeding requiring no specific intervention. Consultation in the oral surgery unit was required following two (2\%) procedures but no intervention was necessary. Specific intervention was required to arrest bleeding which occurred following seven (6\%) procedures. This compares favourably with the notable studies in the literature to date and those reported with VKAs. Gomez-Moreno et al. ${ }^{24}$ reported a series of 29 patients who had dental implant surgery while prescribed dabigatran. Patients had their implants inserted 12 hours after the last dose of dabigatran. Following surgery patients were given gauzes impregnated with $5 \%$ tranexamic acid to bite on for 30-60 minutes. Dabigatran was re-started eight hours after treatment. Two episodes of minor bleeding were reported (7\%) which were managed with additional gauzes impregnated with $5 \%$ tranexamic acid. Another study from the same group of authors ${ }^{25}$ reported on 18 patients who had dental implant surgery while prescribed rivaroxaban therapy, without any interruption or modification of the rivaroxaban regime. Following surgery all patients were given gauzes impregnated with $5 \%$ tranexamic acid to bite on for 30-60 minutes. One patient (5.5\%) presented the day following surgery with moderate bleeding which was successfully managed with gauzes impregnated with $5 \%$ tranexamic acid. In another dental implant surgery setting, Clemm et al. ${ }^{26}$ analysed the post-operative bleeding risk of 117 patients continuing their anticoagulation therapy and undergoing implant surgery and bone grafting procedures. There were four cases of bleeding (3.4\%) in the antithrombotic cohort of 101 patients, with no reports of post-operative bleeding in the 16 patients prescribed DOACs. More recently, Mauprivez and colleagues ${ }^{27}$ have compared the incidence of post-operative bleeding events following dental extractions between patients prescribed DOACs (31 patients) and VKAs (20 patients). Five patients taking DOACs had seven bleeding episodes and four patients taking VKAs had five bleeding episodes. The authors of this study conclude that dental procedures can be performed safely in an outpatient facility in patients prescribed a DOAC, by applying local haemostatic measures, without interrupting or modifying the DOAC. In the studies published to date, local haemostatic measures were applied. In our cohort, local haemostatic measures were similarly applied and positive outcomes were observed in most cases. This demonstrates that in simple cases the DOACs can be safely managed in the context of dentoalveolar surgery, providing careful consideration is given to the timing of the last dose in relation to the surgical procedure and the time of re-commencement of the DOAC. However, for those cases which are complicated by the patient being frail elderly, having relevant medical co-morbidities or taking concomitant anti-platelet therapy, dental surgeons should seek specialist medical advice.

In this new era of oral anticoagulation it is important that the dental surgeon is aware that a patient is taking a DOAC. Early in our study there was one case where the dental surgeon was unaware of the prescription of a DOAC and in this instance the patient experienced bleeding post-operatively. Recently a study conducted in Scotland has highlighted this problem outside of the dental setting, ${ }^{28}$ with the authors demonstrating through survey data a lack of understanding and awareness of these newer anticoagulants and their specific properties among prescribers in both primary and secondary care, including independent nurse and pharmacist prescribers. This highlights the importance of awareness of these agents and that they should command the same respect as warfarin and other VKAs.

The rapid onset of action of the DOACs needs careful consideration in the periprocedural setting. For patients prescribed a once daily DOAC, following advice to take their usual dose in the morning, we aimed to complete the procedure late in the afternoon when the concentration was decreasing, in the knowledge that the next dose of DOAC would not be taken until the following day. However, for those taking twice daily DOAC, conducting a procedure late in the afternoon could lead to the patient taking their evening dose soon after the completion of treatment. Therefore, 
careful consideration should be given to when the DOAC should be re-commenced. Furthermore, patients should be given clear instructions on what to do if they experience bleeding when they get home. In other words, a form of safety netting is required.

In our cohort, 14 patients (15 procedures), did not follow the specific instructions given to them on omitting doses before the procedure, and/or after treatment. We found that patients tended to omit more doses than advised and if not identified this could lead a surgeon into a false sense of security when little or no bleeding occurs. Dental surgeons should be mindful of this. To the best of our knowledge, no patients suffered a pathological thrombotic event as a result of omitting/delaying doses of a DOAC.

Finally, a sizeable number of patients in our cohort were older adults. It is expected that the pharmacokinetic properties of the DOACs are likely to be altered in the very elderly, particularly those classed as frail. Continuing the DOAC as per usual may lead to problems with bleeding and consideration should therefore be given to omitting one or two doses if the clinical situation allows. The majority of patients in our study had their renal function checked in order to assess the potential for accumulation of the DOAC. We found that patients with renal impairment had already had the DOAC dose adjusted to account for this.

Based on our experience, dentists conducting oral surgical procedures might anticipate a post-operative bleeding rate, requiring additional local measures for haemostasis in the region of $6 \%$. Patients at increased risk of bleeding (frail elderly, those with relevant medical complications or on concurrent antiplatelet therapy and those requiring extensive surgical treatment) should be considered for referral to secondary care for specialist input. In these cases, any decision to alter the DOAC regime should be made on a case by case basis, balancing those factors likely to increase the risk of bleeding with the risk of thrombosis.

The results of our study should be considered in the context of its limitations. They are limited by the lack of a control arm and by virtue of being from a single centre. Despite these limitations our report provides an early valuable insight into the practical management of patients prescribed DOACs undergoing oral surgical procedures.

In conclusion, it is possible to safely conduct dentoalveolar procedures for patients prescribed concurrent DOACs. In order to minimise post-operative bleeding and complication, it is important to consider the time the DOAC is usually ingested and when the procedure is planned. Dental surgeons should aim to undertake the procedure when peak DOAC concentrations have subsided ie, 5-6 hours post last dose. Current SDCEP guidance suggests achieving this by delaying the morning dose of a DOAC for procedures with a high risk of bleeding. We endorse this recommendation as it avoids having to treat patients in the late afternoon when there is limited time to manage persistent bleeding. Careful consideration should also be given to when the DOAC is recommenced. We suggest waiting a minimum of four to six hours after the procedure and that haemostasis should be secure. We would also advise that local measures to promote haemostasis, ie, haemostatic packing and soft tissue suturing, are used in every case. Patients at high risk of bleeding, should be considered for referral to specialist units for their procedures. Further clinical experience and controlled cohort studies will determine the optimum periprocedural strategy for managing this growing cohort of patients.

1. Connolly S J, Ezekowitz M D, Yusuf S et al. Dabigatran versus warfarin in patients with atrial fibrillation. New Engl J Med 2009; 361: 1139-1151.

2. Randall C. Surgical management of the primary care dental patient on warfarin. Dent Update 2005; 32: 414-416.

3. Al-Mubarak S, Rass M A, Alsuwyed A, Alabulaaly A, Ciancio $S$. Thromboembolic risk and bleeding in patients maintaining or stopping oral anticoagulant therapy during dental extraction. J Thromb Haemost 2006; 4: 689-691.

4. Randall C. Surgical Management of the Primary Care Dental Patient on Warfarin: North West Medicines Information Centre. 2007. Available online at www.app. dundee.ac.uk/tuith/Static/info/warfarin.pdf (accessed February 2016).

5. Perry D J, Noakes T J C, Helliwell PS. Guidelines for the management of patients on oral anticoagulants requiring dental surgery. Br Dent J 2007; 203: 389-393.

6. National Institute of Health and Care Excellence. Non-vitamin $\mathrm{K}$ antagonist oral anticoagulants (NOACs). Key therapeutic topic, 2016. Available online at http://www. nice.org.uk/advice/ktt16 (accessed February 2016).

7. Baglin T. Clinical use of new oral anticoagulant drugs: dabigatran and rivaroxaban. BJH 2013; 163: 160-167.
8. Hart R G, Eiklboom A J, Ingram A J, Herzog C A. Anticoagulants in atrial fibrillation patients with chronic kidney disease. Nat Rev Neph 2012; 8: 569-578.

9. Beyer-Westendorf J, Förster K, Pannach S et al. Rates, management and outcome of bleeding complications during rivaroxaban therapy in daily care: results from the Dresden NOAC registry. Blood 2014; 124: 955-963.

10. Ageno W, Mantovani L G, Haas S et al. Safety and effectiveness of oral rivaroxaban versus standard anticoagulation for the treatment of symptomatic deep-vein thrombosis (XALIA): an international, prospective, non-interventional study. Lancet Haematology 2015; 3: e12-e21.

11. Camm A J, Amarenco P, Haas $S$ et al., on behalf of the Xantus investigators. XANTUS: a real-world, prospective, observational study of patients treated with rivaroxaban for stroke prevention in atrial fibrillation. Eur Heart J 2015; 37: 1145-1153.

12. Douketis J D, Healey J S, Brueckmann M et al. Urgent surgery or procedure in patients taking dabigatran or warfarin: Analysis if perioperative outcomes from the RELY trial. Thromb Res 2016; 139: 77-81.

13. Kerr R, Ogden G, Sime G. Anticoagulant Guidelines. Br Dent J 2013; 214: 430.

14. Griffiths M, Scully C. New Anticoagulants. Br Dent J 2012; 212: 96.

15. de Mendonca M. Warfarin paradox. Br Dent J 2013; 214: 4-5.

16. Scully C. Anticoagulant update. Br Dent J 2013; 215: 103-104.

17. Fakhri HR, Janket SJ, Jackson E A et al. Tutorial in oral antithrombotic therapy: biology and dental implications. Med Oral Patol Oral Ci Bucal 2013; 18: e461-472.

18. Syyed N, Ansell M, Sood V. Dabigatran (Pradaxa $($ ): surgeon's friend or foe? Br Dent J 2014; 217: 623-626.

19. Breik O, Cheng A, Sambrook P J, Goss A N. Protocol in managing oral surgical patients taking dabigatran. Aus Dent J 2014; 59: 296-301.

20. Johnston S. An evidence summary of the management of patients taking direct oral anticoagulants (DOACs) undergoing dental surgery. Int J Or Max Surg 2016; 45: 618-630.

21. Davis C, Robertson C, Shivkumar S, Lee M. Implications of dabigatran, a direct thrombin inhibitor, for oral surgery practice. J Can Dent Assoc 2013; 79: d74.

22. Scottish Dental Clinical Effectiveness Programme. Management of Dental Patients Taking Anticoagulants or Antiplatelets. 2015. Available online at http:// www.sdcep.org.uk/published-guidance/anticoagulants-and-antiplatelets/ (accessed February 2016).

23. Anon. Novel oral anticoagulants (NOACs) for stroke pre vention in atrial fibrillation. South London Cardiovascular and Stroke Network. Available online at http://www. slcsn.nhs.uk/noacs.html (accessed February 2016).

24. Gomez-Moreno G, Fernandez-Cejas E, Aguilar-Salvatierra $A$ et al. Dental implant surgery in patients in treatment by dabigatran. Clin Oral Implan Res 2016; DOI:10.1111/clr.12785.

25. Gomez-Moreno G, Aguilar-Salvatierra A, Fernandez-Cejas $\mathrm{E}$ et al. Dental implant surgery in patients in treatment with the anticoagulant oral rivaroxaban. Clin Oral Implan Res 2015; DOI:10.1111/clr.12653.

26. Clemm R, Neukam F W, Rusche B et al. Management of anticoagulated patients in implant therapy: a clinical comparative study. Clin Oral Implan Res 2015; 27: 1274-1282.

27. Mauprivez C, Khonsari R H, Razouk O, Goudot P, Lesclous P, Descroix V. Management of dental extraction in patients undergoing anticoagulant oral direct treatment - a pilot study. Oral Sur, Oral Med, Oral Path, Oral Radio 2016: 122: e146-e155.

28. Olaiya A, Lurie B, Watt B, Mc Donald L, Greaves M, Watson $\mathrm{H} \mathrm{G}$. An observational study of direct oral anticoagulant awareness indicating recognition with potential for patient harm. J Thromb Haem 2016; 14: 987-990. 